\title{
Materials Horizons
}

Check for updates

Cite this: Mater. Horiz., 2022, 9, 500

Received 22nd August 2021 , Accepted 19th November 2021

DOI: 10.1039/d1mh01357b

rsc.li/materials-horizons

\section{Backbone-driven host-dopant miscibility modulates molecular doping in NDI conjugated polymers $\dagger$}

\author{
Diego Rosas Villalva, (D) a Saumya Singh, ${ }^{b}$ Luke A. Galuska, (D) Anirudh Sharma, (D) \\ Jianhua Han, ${ }^{a}$ Jian Liu, ${ }^{d}$ Md Azimul Haque, (D) a Soyeong Jang, ${ }^{a}$ \\ Abdul Hamid Emwas, ${ }^{e}$ L. Jan Anton Koster, (D) ${ }^{d}$ Xiaodan Gu, (iD c \\ Bob C. Schroeder (D) *b and Derya Baran (D)*a
}

Molecular doping is the key to enabling organic electronic devices, however, the design strategies to maximize doping efficiency demands further clarity and comprehension. Previous reports focus on the effect of the side chains, but the role of the backbone is still not well understood. In this study, we synthesize a series of NDI-based copolymers with bithiophene, vinylene, and acetylenic moieties (P1G, P2G, and P3G, respectively), all containing branched triethylene glycol side chains. Using computational and experimental methods, we explore the impact of the conjugated backbone using three key parameters for doping in organic semiconductors: energy levels, microstructure, and miscibility. Our experimental results show that P1G undergoes the most efficient n-type doping owed primarily to its higher dipole moment, and better host-dopant miscibility with $\mathrm{N}$ DMBI. In contrast, P2G and P3G possess more planar backbones than P1G, but the lack of long-range order, and poor host-dopant miscibility limit their doping efficiency. Our data suggest that backbone planarity alone is not enough to maximize the electrical conductivity $(\sigma)$ of n-type doped organic semiconductors, and that backbone polarity also plays an important role in enhancing $\sigma$ via host-dopant miscibility. Finally, the thermoelectric properties of doped P1G exhibit a power factor of $0.077 \mu \mathrm{W} \mathrm{m} \mathrm{m}^{-1} \mathrm{~K}^{-2}$, and ultra-low in-plane thermal conductivity of $0.13 \mathrm{~W} \mathrm{~m}^{-1} \mathrm{~K}^{-1}$ at $5 \mathrm{~mol} \%$ of $\mathrm{N}-\mathrm{DMBI}$, which is among the lowest thermal conductivity values reported for $n$-type doped conjugated polymers.

\section{Introduction}

Organic semiconductors (OSCs) hold the potential to enable a whole new generation of electronics such as flexible and

\section{New concepts}

In recent studies, modulation of host-dopant miscibility led to enhanced molecular doping of organic semiconductors (OSCs), thus enabling their applicability several organic electronic devices such as organic thermoelectrics. The most common approach includes the addition of oligoethylene glycol side chains to the OSC to improve their miscibility with polar dopants such as N-DMBI. Herein we focus on the conjugated polymer (CP) backbone and its impact on the molecular doping efficiency of naphthalene diimide (NDI) based CPs, with various degrees of backbone planarity, rigidity, and dipole moments. Our data show a strong correlation between the backbone dipole moment and the maximum value of the electrical conductivity achieved upon molecular doping with N-DMBI. The most polar backbone in the series (P1G) showed the highest electrical conductivity at the lowest doping concentrations. On the contrary, P3G possessing the least polar backbone showed the lowest electrical conductivity, requiring larger dopant amounts to reach its maximum conductivity. This suggests a backbone-driven miscibility effect that can enable or limit molecular doping efficiency. Consequently, we measured the temperaturedependent thermoelectric properties of P1G, finding an ultra-low inplane thermal conductivity value of $0.13 \mathrm{~W} \mathrm{~m}^{-1} \mathrm{~K}^{-1}$ enabled by the branched side chains. This opens a new possible strategy to further decrease the thermal conductivity of CPs.

wearable devices. ${ }^{1}$ Unlike their inorganic counterparts, OSCs can be solution-processed and possess inherent flexibility. ${ }^{2,3}$ However, they are still limited by their low mobility. Molecular doping has become a major enabler of several technologies such as transport layers in organic photovoltaics and organic light-emitting diodes, organic photodiodes, organic field-effect transistors, and most recently in organic electrochemical

\footnotetext{
${ }^{a}$ King Abdullah University of Science and Technology (KAUST), Physical Sciences and Engineering Division (PSE), KAUST Solar Center (KSC), Thuwal 23955, Saudi Arabia. E-mail: derya.baran@kaust.edu.sa

${ }^{b}$ Department of Chemistry, University College London, 20 Gordon Street, London, WC1H OAJ, UK. E-mail: b.c.schroeder@ucl.ac.uk

${ }^{c}$ School of Polymer Science and Engineering, University of Southern Mississippi, Hattiesburg, MS, 39406, USA

${ }^{d}$ Zernike Institute for Advanced Materials, University of Groningen, Nijenborgh 4, 9747 AG, Groningen, The Netherlands

${ }^{e}$ King Abdullah University of Science and Technology (KAUST), Core Labs, Thuwal 23955, Saudi Arabia

$\dagger$ Electronic supplementary information (ESI) available. See DOI: 10.1039/d1mh01357b
} 
transistors and organic thermoelectrics (OTEs). ${ }^{4-11}$ While p-type doped OSCs have achieved $\sigma$ in a range of $10^{3}$ to $10^{4} \mathrm{~S} \mathrm{~cm}^{-1}$, n-type doped OSCs possess $\sigma$ in the order of $10^{0}$ to $10^{1} \mathrm{~S} \mathrm{~cm}^{-1} \cdot{ }^{12-16}$ Functional organic thermoelectric devices require both n-type and p-type materials. Hence, the design of new n-type conjugated polymers (CPs) and, the understanding of the rules to design them, is of uttermost importance to overcome these challenges. ${ }^{17-19}$

Naphthalenediimide (NDI) based n-type OSCs have been explored, mainly through modular modification of P(NDIOD2T) (N2200), such as side chain alterations to increase dopant miscibility and enhance electrical conductivity. ${ }^{20-22}$ Additionally, the dipole moment of polar side chains screens the backbone and dopant charges, thus reducing Coulombic binding and improving the fraction of mobile charge carriers. ${ }^{23}$ Other design approaches include backbone modification such as substitution of bithiophene (2T) units by bithiazole. ${ }^{24,25}$ These modifications increased the backbone planarity, leading to better charge delocalization along the polymer backbone and enhanced molecular packing for better charge transport. Further modifications include backbone halogenation which can induce deeper LUMO levels to improve doping efficiency, and introducing rigid bonds to increase the backbone planarity, improve film crystallinity, and provide enhanced charge transport properties. ${ }^{26,27}$

In this contribution, we explore the backbone modification of NDI-based CPs and its effect on molecular doping.
The synthesized polymers substituted with bithiophene, vinylene, and acetylenic groups, are named P(NDI4TEG-2T), P(NDI4TEG-V), and $\mathrm{P}$ (NDI4TEG-A) respectively. The synthetic route and chemical structure of the three polymers is depicted in Scheme 1. For simplicity, these will be referred to as P1G, P2G, and P3G respectively throughout the text. All the synthesized CPs contain branched TEG side chains motivated from previous reports to improve host-dopant miscibility and reduce Coulomb interaction, allowing to study the effect of the backbone on molecular doping and the electronic properties of CPs. ${ }^{20,21,28}$

Experimental results showed that all three polymers can be doped by N-DMBI, however, the conductivity of P1G outperformed the other two polymers owed to a higher crystallinity and good miscibility with N-DMBI. While P2G exhibits good miscibility with $\mathrm{N}$-DMBI, the lack of long-range order limits its $\sigma$. P3G shows poor host-dopant miscibility and a low degree of molecular order, which make it the polymer with the lowest $\sigma$. Finally, we analysed the temperature-dependent thermoelectric properties of P1G as its electrical conductivity is the largest of the three CPs. Our results show that OTEs can benefit from branched TEG side chains, as only $5 \mathrm{~mol} \%$ of $\mathrm{N}$-DMBI suffices to maximize the power factor (PF). Remarkably, P1G shows an in-plane thermal conductivity $\left(\kappa_{\|}\right)$of only $0.13 \mathrm{~W} \mathrm{~m}^{-1} \mathrm{~K}^{-1}$, one of the lowest reported values for CPs. This suggests that branched TEG side chains are a promising strategy to reduce the thermal conductivity of CPs, in addition to improving their miscibility with polar dopants.
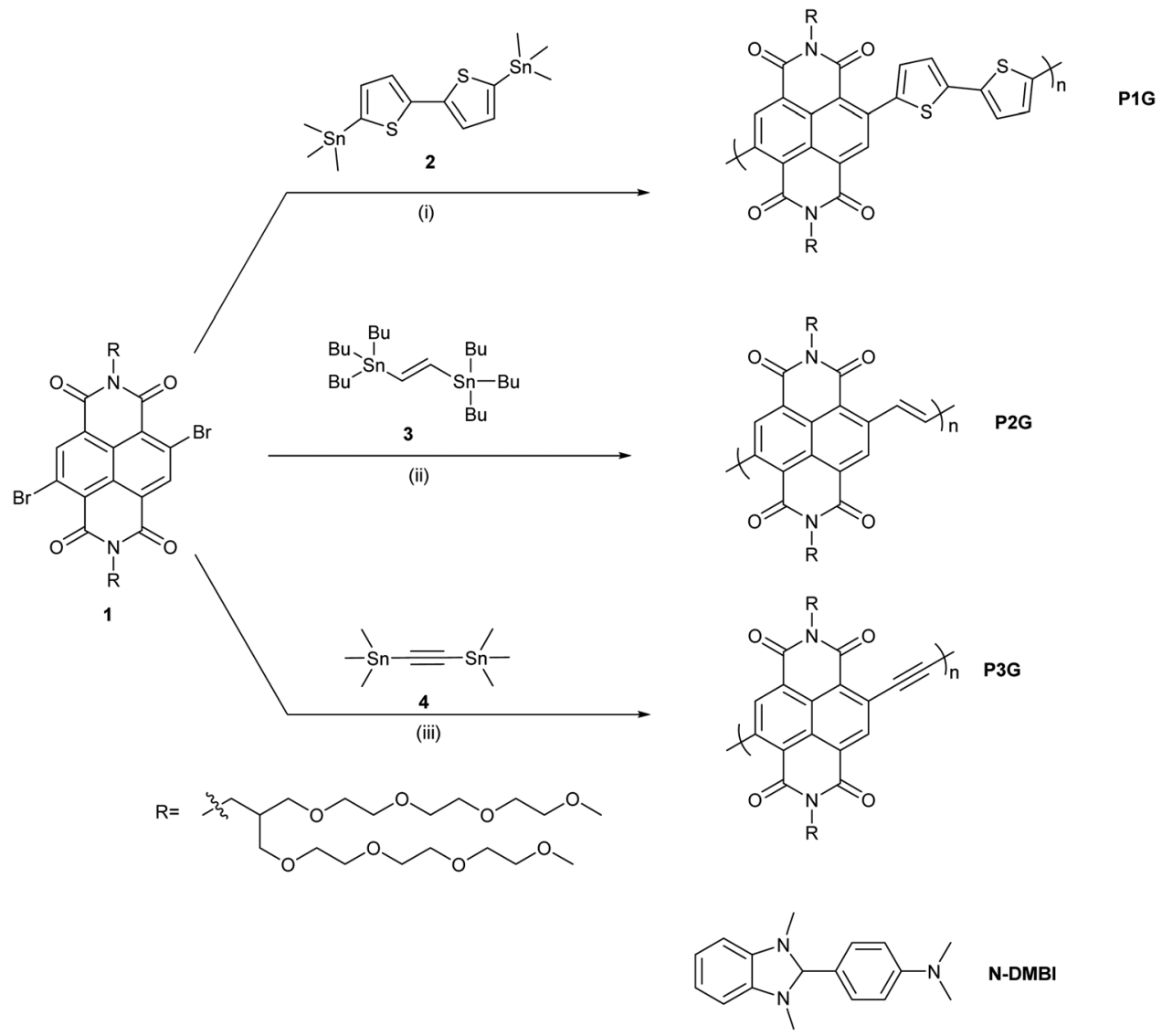

Scheme 1 Synthetic routes towards polymers P1G, P2G and P3G, and chemical structure of N-DMBI. 


\section{Results and discussion}

Copolymers were synthesized via Stille coupling polymerization in chlorobenzene (Scheme 1). Synthesis of NDI monomer 1 and polymer $\mathrm{P} 2 \mathrm{G}$ is reported in our previous work and synthetic protocols for polymers $\mathrm{P} 1 \mathrm{G}$ and $\mathrm{P} 3 \mathrm{G}$ are described in the supporting information. ${ }^{29}$ The molecular weight analysis of the polymers P1G $\left(M_{\mathrm{n}}=21.4 \mathrm{~kg} \mathrm{~mol}^{-1}, Ð=1.5\right)$ and $\mathrm{P} 2 \mathrm{G}$ $\left(M_{\mathrm{n}}=6.9 \mathrm{~kg} \mathrm{~mol}{ }^{-1}, D=1.8\right)$ was carried out by hightemperature gel permeation chromatography (GPC) using dichlorobenzene as a solvent. The molecular weight of P3G could not be obtained by GPC analysis due to the strong aggregation of P3G in solution, owing to its planar backbone.

Previous reports suggest that the bithiophene-induced backbone twist in N2200 and perylene dimiide-bithiophene CPs limits the polaron delocalization length and the electrical conductivity, showing that more planar backbones possess higher $\sigma .^{24,30}$ This makes backbone planarization an attractive approach to improve $\sigma$ of n-type doped CPs. Hence, the designs of P2G and P3G are aimed at increasing the backbone planarity. Previous reports have used non-bonding conformational locks to improve molecular packing and charge transport properties. ${ }^{31-33}$ To better understand the role of the polymer conjugated backbone on molecular doping, we considered three parameters. First, the energetics of the polymers (considering lowest unoccupied molecular orbitals (LUMO)), second, the backbone planarity and rigidity, and third, the dipole moment of the backbone, which we shall define as the local charge separation induced by the atoms within the backbone of a polymer while disregarding the contributions of the atoms in the side chains.

The energy levels of the frontier molecular orbitals for the tetramers of the CPs were calculated by DFT (Fig. S4, ESI $\dagger$ ), the energy levels are represented in Fig. 1a. P1G shows the shallowest LUMO $(-3.46 \mathrm{eV})$ followed by P2G $(-3.72 \mathrm{eV})$ and P3G $(-3.98 \mathrm{eV})$, suggesting that all three polymers should be efficiently doped by the singly occupied molecular orbital (SOMO) of N-DMBI at $-2.36 \mathrm{eV}$ (Fig. S5, ESI $\dagger$ ). For P1G, the HOMO is mainly delocalized on the bithiophene donor subunit, and the LUMO is delocalized on the NDI acceptor subunit, similarly to previously reported D-A CPs. ${ }^{34}$ In contrast, P2G and P3G show both HOMO and LUMO delocalized along the whole tetramer backbone, such delocalization might be beneficial for charge transport by extending the probability of both intra- and intermolecular hopping, consequently improve $\sigma .^{35,36}$ Additionally, we calculated the torsion potential for all three polymers (Fig. 1b). P1G showed a twisted backbone with an angle of $\sim 48^{\circ}$ for the bond between the NDI and $2 \mathrm{~T}$ subunit, along with a moderate torsion energy barrier of $0.13 \mathrm{eV}$. P2G showed a more planar backbone with an angle of $\sim 25^{\circ}$ between NDI and vinylene subunits, it also possesses the largest torsion energy barrier $(0.27 \mathrm{eV})$ making it the most rigid backbone of the three CPs, owing to the interaction of the carbonyl group in the NDI and the hydrogen of the vinylene which work as a non-bonding conformational lock. ${ }^{32,37}$ P3G shows the most planar backbone, a)

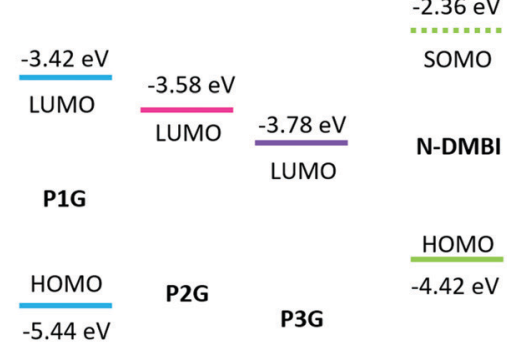

$\frac{\text { HOMO }}{-6.45 \mathrm{eV}} \frac{\text { HOMO }}{-6.53 \mathrm{eV}}$

c)

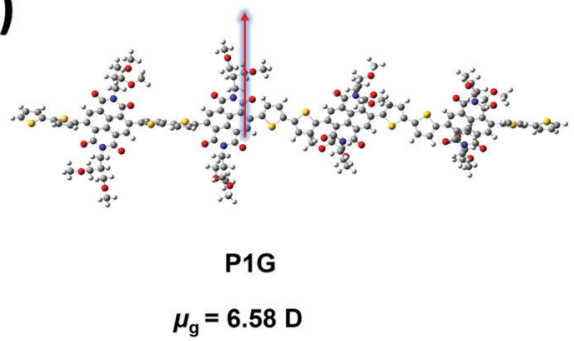

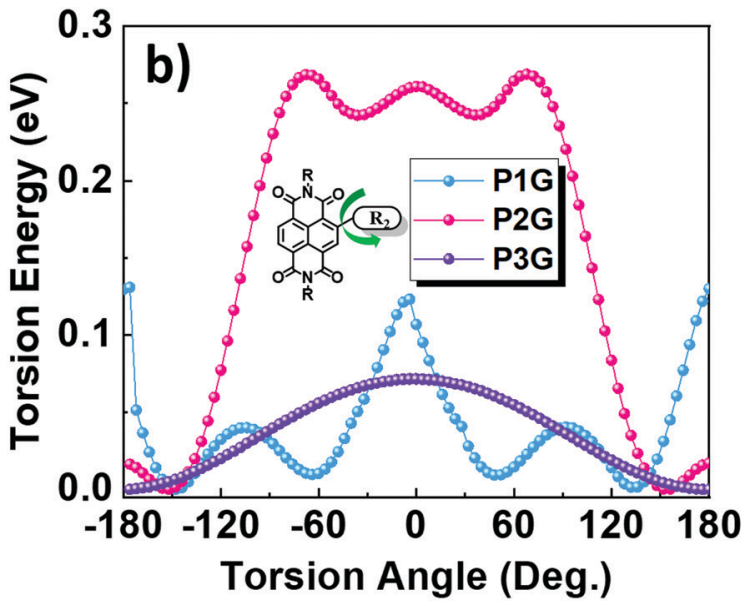

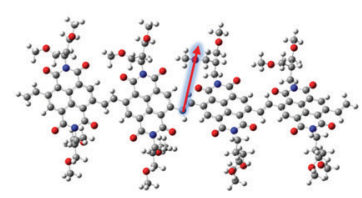

P2G

$\mu_{\mathrm{g}}=3.13 \mathrm{D}$

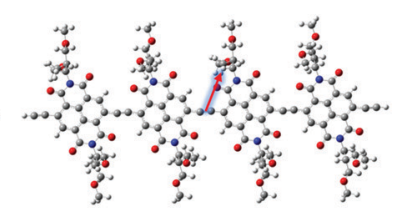

P3G

$\mu_{\mathrm{g}}=1.50 \mathrm{D}$

Fig. 1 Results from DFT calculations. (a) energy levels of CPs and N-DMBI (b) torsion energy profiles of the NDI-R $\mathrm{R}_{2}$ bond. (c) Energy minimized 3D structure and dipole moments of the backbone of tetramer models for CPs, arrows are vectors depicting the magnitude and direction of the dipole moment. 
stabilizing $\sim 0^{\circ}$, however, its rather low torsion energy barrier $(0.07 \mathrm{eV})$ implies that several conformers might be possible for this CP and hinder its charge transport properties. Last, we computed the dipole moments of the tetramers through DFT (Fig. 1c). The arrows are vectors depicting the magnitude and direction of the backbone dipole moment with respect to the conjugated backbone. P1G shows the largest dipole moment of 6.58 D, followed by P2G with 3.13 D, and P3G has the smallest dipole of 1.50 D. Although these CPs contain TEG side chains, we are interested in observing if the CP backbone will still influence the miscibility with the dopant, and consequently its electronic properties. N-DMBI, being a polar molecule, should have better miscibility with P1G due to its larger dipole moment.

After carrying out the DFT calculations, we proceeded to characterize the CP films. Pristine and doped polymer films were spin-coated from stock solutions in chloroform with a concentration of $10 \mathrm{mg} \mathrm{mL} \mathrm{m}^{-1}$. Solution doping was performed by mixing aliquots of stock chloroform solutions of polymer and N-DMBI dopant at corresponding amounts to achieve the desired molar ratio. This was calculated by considering the ratio of dopant molecules to repeat units of each polymer. Fig. S6 (ESI $\dagger$ ) shows the absorption spectra of the pristine and doped polymer films. P1G shows similar absorption features compared to other N2200 analogue copolymers, with an intramolecular charge transfer (ICT) peak at $\sim 725 \mathrm{~nm} \cdot{ }^{38}$ Addition of
$\mathrm{N}$-DMBI resulted in the bleaching of this peak as a result of the charge transfer from N-DMBI to P1G. On the other hand, P2G and P3G lack the ICT feature and show the peaks of the $\pi-\pi^{*}$ transition at 640 and $555 \mathrm{~nm}$, respectively. Addition of the $\mathrm{N}$-DMBI results in bleaching of the $\pi-\pi^{*}$ transition and formation of new spectral features in the UV-vis spectrum with peaks in $\sim 690 \mathrm{~nm}(\mathrm{P} 2 \mathrm{G})$ and $665 \mathrm{~nm}$ (P3G). An additional broadband feature appears in the near-infrared region for both polymers. This set of features can be correlated to the polaronic and bipolaronic transitions showing successful reduction of P2G and P3G by N-DMBI.

The electrical conductivity of pristine and doped polymers was compared by spin coating films on top of glass substrates with pre-patterned gold electrodes (Fig. 2a-c). All samples showed improved $\sigma$ upon addition of N-DMBI, however, this change is more significant in the case of P1G, where $\sigma$ of the doped films is about three orders of magnitude higher compared to neat polymer. In the case of $\mathrm{P} 2 \mathrm{G}$ and $\mathrm{P} 3 \mathrm{G}$, the increment was about two orders of magnitude for each polymer. Additionally, $\mathrm{P} 1 \mathrm{G}$ reached its maximum $\sigma$ at rather low dopant concentrations, i.e. 10 to 15 mol\% N-DMBI. The addition of higher dopant concentrations led to a reduction of $\sigma$, possibly due to a decrease in mobility $(\mu)$ originated by carriercarrier scattering. ${ }^{39}$ In contrast P2G needed $25 \mathrm{~mol} \%$, and P3G more than $30 \mathrm{~mol} \%$ dopant concentration to achieve their maximum conductivity. Further increasing the dopant ratio
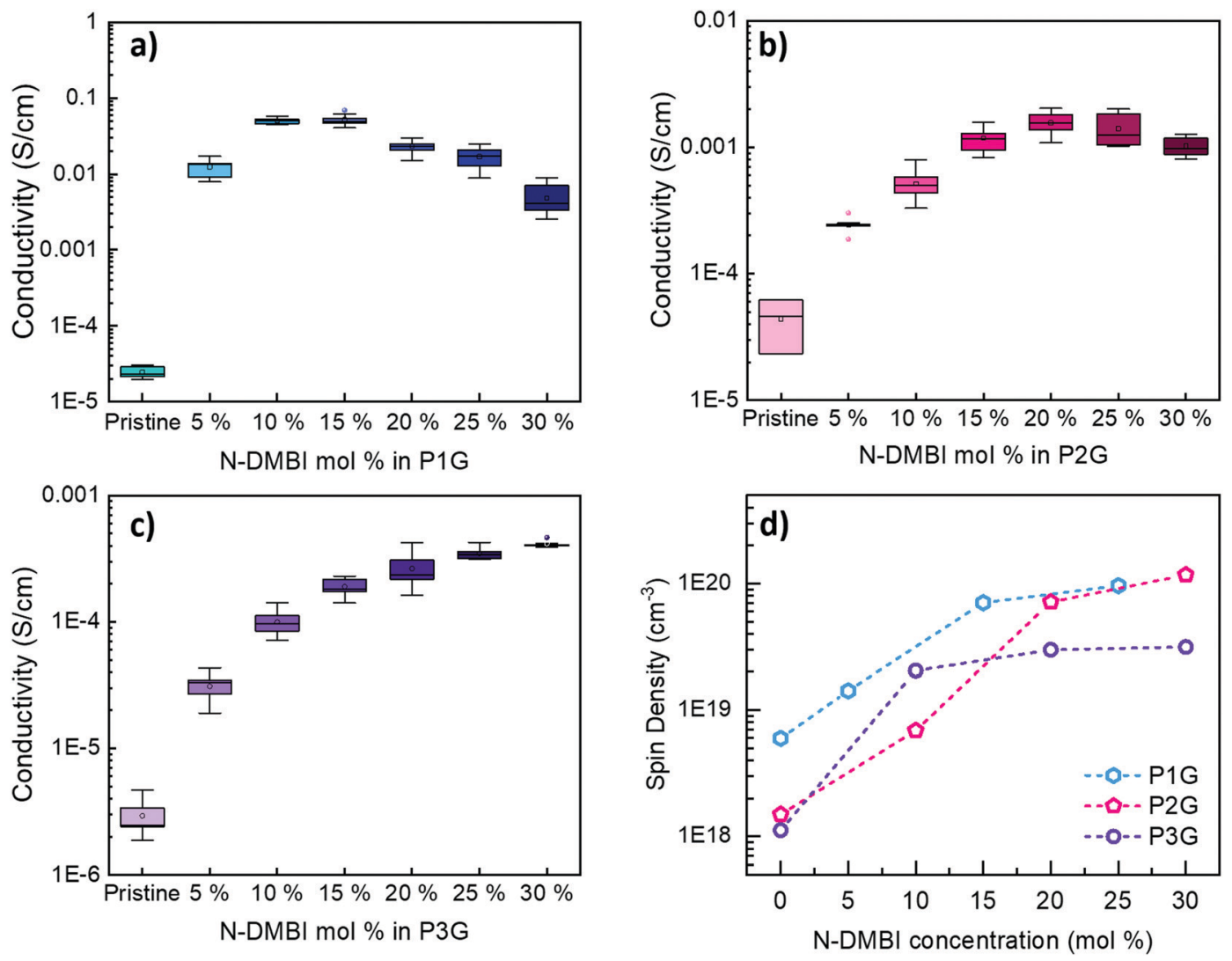

Fig. $2 \sigma$ of P1G (a), P2G (b), and P3G (c) films doped with N-DMBI. Representative I-V curves are shown in Fig. S7 (ESI $\dagger$ ). (d) Shows a summary of the spin densities obtained from EPR. 
for P3G proved too challenging owed to the poor solubility of P3G, which limited the formation of continuous films and the accurate measurement of $\sigma$. These observations correlate with the calculated dipole moments. The polymer with the largest dipole moment (P1G) requires the least amount of N-DMBI to reach its maximum conductivity, and the one with the smallest dipole moment (P3G) requires more than twice the amount to achieve its maximum conductivity. Previous studies in fullerene systems have shown that increasing host-dopant miscibility can decrease the amount of dopant needed to achieve maximum conductivity. ${ }^{40}$ We further investigated these phenomena using AFM topography and phase imaging. In the case of P1G and P2G (Fig. S8 and S9, ESI $\dagger$ ), no dopant clustering appeared, even at high doping concentrations. In the case of P3G, the topography images (Fig. S10, ESI $\dagger$ ) reveal the formation of several aggregates only present in the doped polymer films. These aggregates show a strong contrast in the AFM phase image indicating such clusters are formed by phase-segregated dopant and implying poor host-dopant miscibility. Such phase segregation explains in part the much lower conductivity observed in P3G. Additionally, we measured the contact angle between water and the undoped CPs, N-DMBI, as well as the blended host-dopant polymer films, to determine their hydrophobic properties. Results are shown in Fig. S11 (ESI $\dagger$ ). P1G and $\mathrm{P} 2 \mathrm{G}$ show values of $54.1^{\circ}$ and $31.6^{\circ}$ respectively. These values are closer to the contact angle of N-DMBI $\left(41.6^{\circ}\right)$ than that of P3G $\left(64.9^{\circ}\right)$ suggesting better miscibility in the systems P1G:N-DMBI and P2G:N-DMBI, than in P3G:N-DMBI. This further confirms the observations carried out in AFM. Finally, the addition of N-DMBI to either P1G or P2G resulted in a decrease of the contact angle, whereas P3G remained unchanged.

To further elaborate on the differences in electronic properties of the three CPs, we proceeded to perform advanced spectroscopic analysis. Electron paramagnetic resonance (EPR) provides a quantitative analysis of unpaired electrons in the form of radical anions within a material, which can be correlated to the charge carrier density and offer an understanding of the differences observed in $\sigma$. We performed EPR in pristine and doped films of the NDI copolymers, the resulting spectra are shown in Fig. S12a-c (ESI $\dagger$ ). The undoped polymer films show very weak EPR signals. The addition of N-DMBI leads to the generation of radical anions and thus large increments of the signal intensity in all polymers. Quantitative analysis of the signals provided the spin density of the samples (Fig. 2d). We observe that the undoped P1G film has a larger spin density $\left(5 \times 10^{18} \mathrm{~cm}^{-3}\right)$ than the undoped films of P2G and P3G $\left(1 \times 10^{18} \mathrm{~cm}^{-3}\right)$. As N-DMBI is added to the samples, the spin density increases, and both P1G and P2G show spin densities of about $1 \times 10^{20} \mathrm{~cm}^{-3}$ while P3G shows only $3 \times 10^{19} \mathrm{~cm}^{-3}$. Although this partially explains the lower $\sigma$ of P3G, it does not explain the difference in $\sigma$ of P1G and P2G at their respective maximums.

The ionization energy (IE) and electron affinity (EA) of all polymers P1G, P2G, and P3G were directly measured on thin films using ultraviolet photoelectron spectroscopy (UPS) and low-energy inverse photoelectron spectroscopy (LE-IPES) (Fig. 3a and $\mathrm{b}$ respectively). The IE and EA of P1G and P2G were found to be $5.58 \mathrm{eV} / 3.62 \mathrm{eV}$ and $5.67 / 3.38 \mathrm{eV}$, respectively. Thin films of P3G were found to have an IE of $5.41 \mathrm{eV}$ and the deepest EA of $3.73 \mathrm{eV}$ among all three polymers. It must be noted that the DFT calculations estimated the orbital energies of tetramers, whereas the spectroscopic studies were carried out in thin solid films of polymers. The orbital energies of the isolated molecules and those in thin solid films are different, ${ }^{41}$ and would explain the differences in the calculated HOMO/ LUMO and the measured IE/EA values. Among all the three polymers, P1G was found to have the most prominent peak at the onset of both UPS and IPES spectra, corresponding to the density of states (Fig. 3a and b) in the IE and EA, respectively. We attribute these features to an additional density of states (DOS) introduced by the ICT between the NDI and 2T subunits of P1G. These features can also be correlated to the peak at $725 \mathrm{~nm}$ in the absorption spectrum of P1G. ${ }^{38}$ Since the electronic states in the IE and EA regions contribute to the electrical conductivity of the materials, $\mathrm{P} 1 \mathrm{G}$ is expected to have the
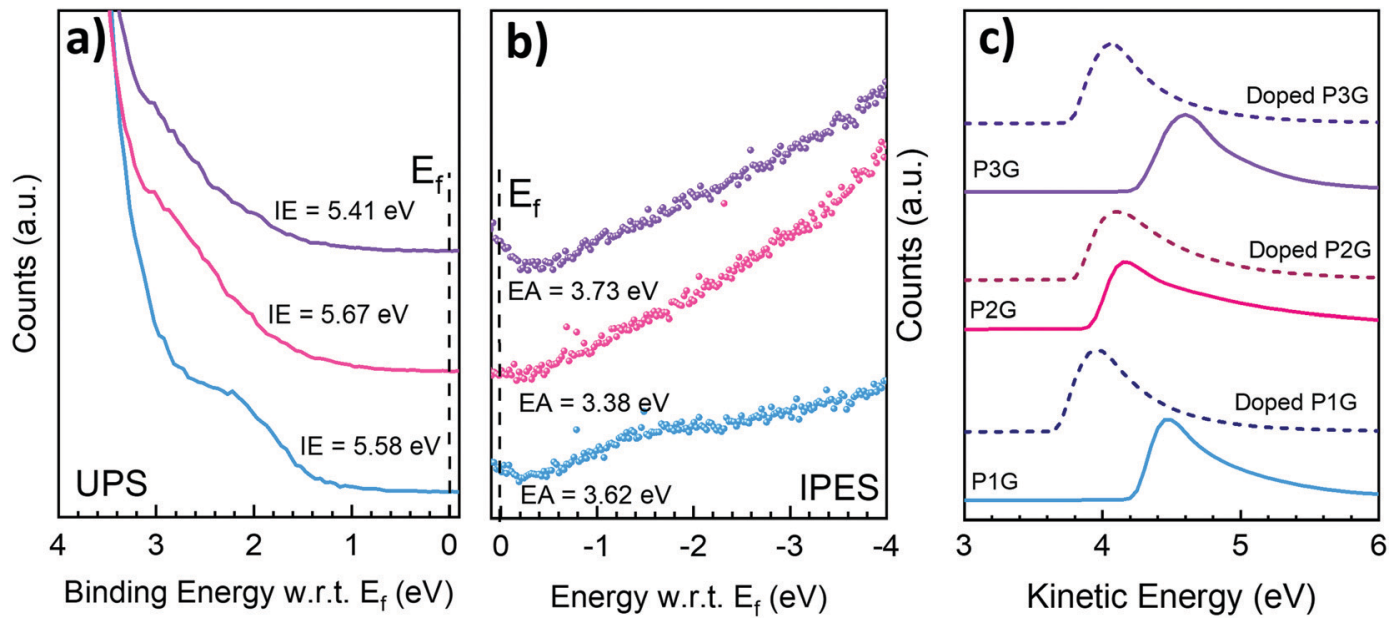

Fig. 3 Photoelectron spectroscopy of the CPs. (a) shows the IE, (b) corresponds to the EA. (c) SECO showing the work function of the undoped films (continuous lines) and their shift upon doping (dashed lines). 
highest electrical conductivity among all the polymers. These features are significantly weaker in the case of P2G and P3G and explain in part their lower $\sigma$.

The secondary electron cut-off (SECO) of the UPS spectra displayed in Fig. 3c, provides direct observation of the surface work function of pristine and doped polymer films. Upon n-type doping, the SECO shifts toward vacuum level. The film of P1G demonstrates a significant reduction of over $0.56 \mathrm{eV}$ in the work function from $4.23 \mathrm{eV}$ to $3.67 \mathrm{eV}$, upon the addition of 20 mol\% N-DMBI. Fig. S13a (ESI $\dagger$ ) shows the IE from the UPS spectra, depicting the shift of $0.3 \mathrm{eV}$ in the IE towards higher binding energy (Fermi level moves away from IE), confirming n-type doping of P1G with N-DMBI. Undoped P2G was found to have a low work function of $3.91 \mathrm{eV}$, which reduced further to $3.8 \mathrm{eV}$ with the addition of N-DMBI. The ability of undoped P2G to modify the work function of a range of electrode surfaces enabled its use as an efficient electron transport layer in organic solar cells. ${ }^{29}$ The IE of P2G demonstrated a small shift of only $0.09 \mathrm{eV}$ towards higher binding energy (Fig. S13b, ESI $\dagger$ ) with $20 \mathrm{~mol} \%$ of N-DMBI, again confirming n-type doping of $\mathrm{P} 2 \mathrm{G}$. Finally, the work function of $\mathrm{P} 3 \mathrm{G}$ was also found to significantly reduce from $4.25 \mathrm{eV}$ to $3.76 \mathrm{eV}$, upon the addition of $20 \mathrm{~mol} \% \mathrm{~N}$-DMBI accompanied by a $0.45 \mathrm{eV}$ shift of the IE towards higher binding energy (Fig. S13c, ESI $\dagger$ ), confirming ntype doping. The IE of P2G (5.39 eV) and P3G (5.63 eV) was found to be largely unchanged with the N-DMBI doping, whereas the IE of P1G was reduced from $5.58 \mathrm{eV}$ to $5.32 \mathrm{eV}$. This shows that P1G undergoes the most efficient doping of the three polymers.

GIWAXS was carried out to study the morphology of the CP films. The 2D data and linecuts are displayed in Fig. 4, (see
Fig. S14 for all 2D GIWAXS scattering results and Fig. S15 for their linecuts, ESI $\dagger$ ) and a summary of the peaks are displayed in Tables S1 and S2 (ESI $\dagger$ ). P1G (Fig. 4a) shows a clear face-on orientation, which is similar to that of N2200, with the lamellar and backbone scattering appearing along the in-plane direction, whereas the $\pi-\pi$ stacking appears along the out-of-plane direction. P2G and P3G on the other hand have an amorphous nature, displaying isotropic diffused rings (Fig. $4 \mathrm{~b}$ and $\mathrm{c}$ respectively). Despite the higher planarity of P3G and higher rigidity of $\mathrm{P} 2 \mathrm{G}$, their films did not develop long-range ordering, leading to lower electrical conductivities. The linecuts in Fig. 4d and e showcase the higher degree of molecular packing for $\mathrm{P} 1 \mathrm{G}$, e.g., prominent (200) scattering peaks at $q=0.5 \mathrm{~A}^{-1}$, compared to P2G and P3G. The addition of N-DMBI did not produce major changes on the $d$-spacing of either P1G or P2G, showing good miscibility with N-DMBI in the amorphous region of the film. Lastly, P3G did not undergo major $d$-spacing changes either, but additional diffraction peaks appeared on the heavily doped samples suggesting strong phase segregation and formation of dopant crystalline domains, which explains why P3G has the lowest electrical conductivity amongst others.

\section{Thermoelectric properties of P1G}

P1G shows higher $\sigma$ at very low dopant loading concentrations suggesting its suitability for thermoelectric applications. We measured its temperature-dependent thermoelectric (TE) properties with a chip-based technique. $\sigma$ and Seebeck coefficient $(\alpha)$ were measured using Van der Pauw configuration to measure temperature-dependent $\sigma$ and $\alpha$; temperaturedependent $\kappa_{\|}$was measured in the same chip using $3 \omega$ method. In the case of $\mathrm{P} 2 \mathrm{G}$ and $\mathrm{P} 3 \mathrm{G}, \sigma$ is lower than the measurement

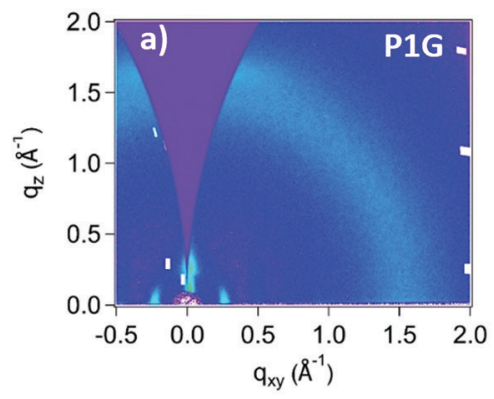

d)

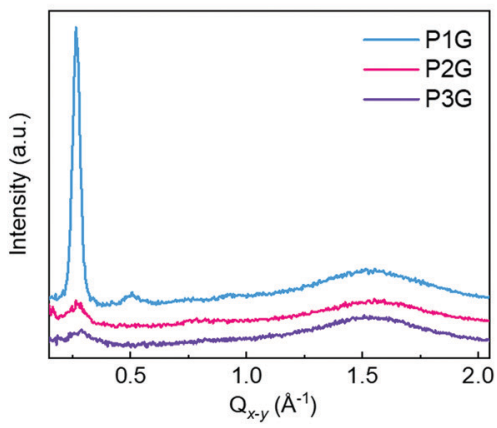

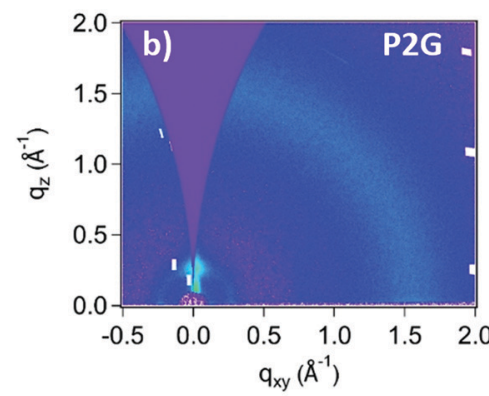

e)

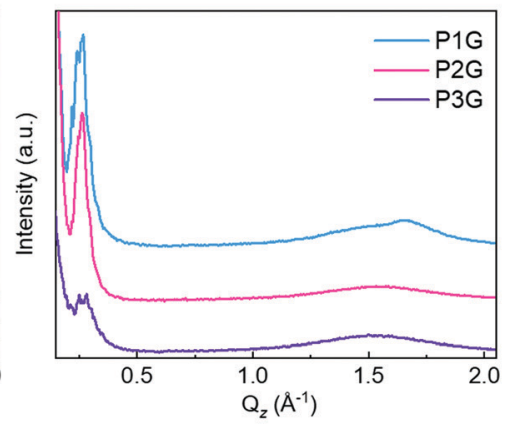

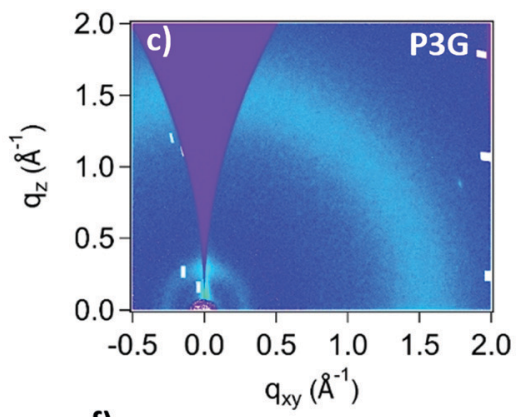

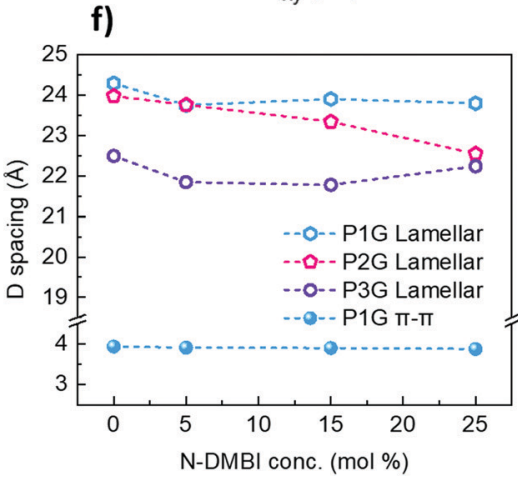

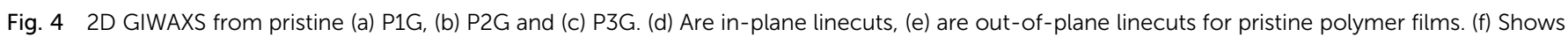
the change of $d$-spacing upon the addition of N-DMBI. 
a)

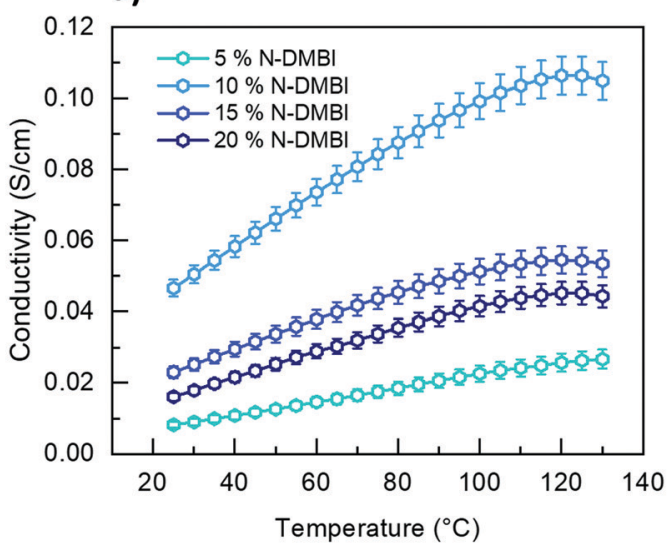

c)

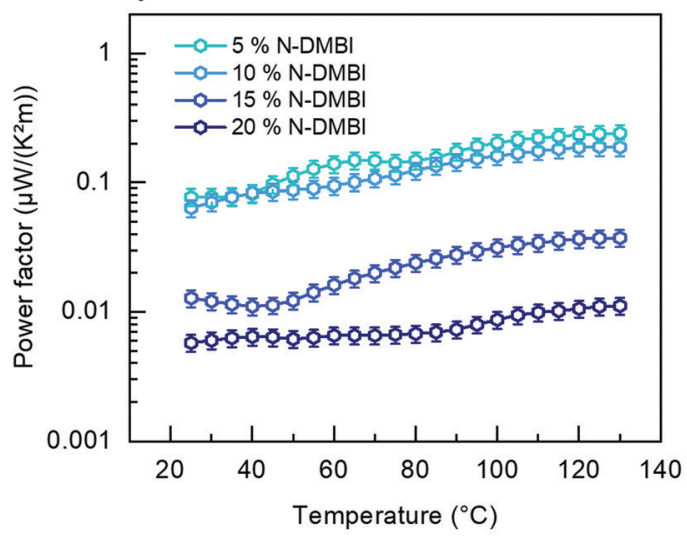

b)

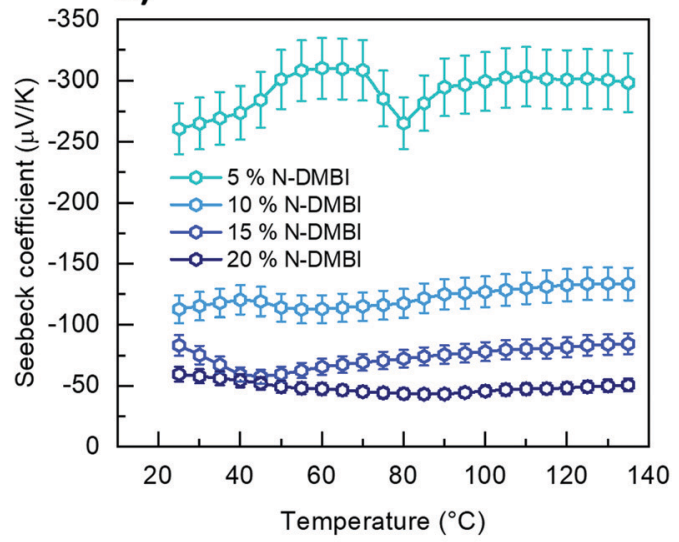

d)

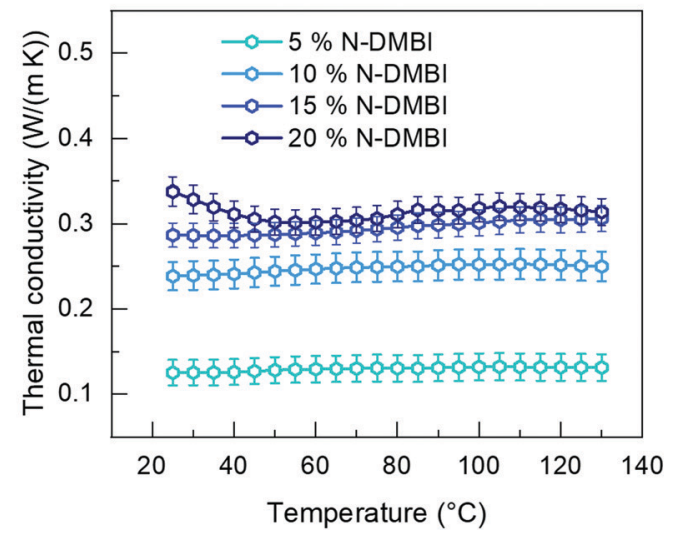

Fig. 5 Temperature-dependent thermoelectric parameter of P1G (a) $\sigma$, (b) $\alpha$, (c) PF, and (d) $\kappa_{\|}$.

limit of the instrument. The temperature-dependent $\sigma$ (Fig. 5a) shows that P1G follows typical semiconductor behaviour i.e. increasing proportionally with temperature. The highest $\alpha$ was measured at $5 \mathrm{~mol} \% \mathrm{~N}$-DMBI, at higher dopant concentrations this value decreases rapidly, as increased dopant concentrations reduce the average energy of the charge carriers, also known as transport energy $\left(E_{\mathrm{t}}\right){ }^{42} \alpha$ shows negligible dependency on the temperature. The highest power factor for P1G was also achieved at this doping concentration $\left(0.077 \mu \mathrm{W} \mathrm{K} \mathrm{K}^{-2} \mathrm{~m}^{-1}\right.$ at room temperature and $0.25 \mu \mathrm{W} \mathrm{K} \mathrm{K}^{-2} \mathrm{~m}^{-1}$ at $130{ }^{\circ} \mathrm{C}$ ). Finally, P1G shows an ultralow $\kappa_{\|}$of $0.13 \mathrm{~W} \mathrm{~m}^{-1} \mathrm{~K}^{-1}$, which is one of the lowest values known for CPs so far. N2200, the alkyl-substituted analogue of P1G, possesses an in-plane thermal conductivity of $0.27 \mathrm{~W} \mathrm{~m}{ }^{-1} \mathrm{~K}^{-1}{ }^{43}$ $\kappa_{\|}$of P1G shows a low dependency on temperature within the studied temperature window. However, increasing the dopant concentration led to increased $\kappa_{\|}$, hence, the highest figure of merit (ZT) of $2 \times 10^{-4}$ was achieved at 5 mol\% of N-DMBI (Fig. S16, ESI $\dagger$ ). This suggests that TEG side chains are a promising approach to reduce the thermal conductivity of CPs, a key factor to improve their figure of merit for thermoelectric applications. ${ }^{44}$

\section{Conclusions}

A series of NDI-based copolymers with branched TEG side chains were synthesized, proposing conjugated vinylene and acetylenic linkers to reduce steric hindrance and improve backbone planarity and rigidity. While these spacer groups can effectively produce planar backbones, only P1G results in oriented molecular packing, P2G and P3G on the other hand are mostly amorphous suggesting backbone planarization alone is not enough to produce long-range ordering. While ntype molecular doping was demonstrated for all three polymers, P1G outperformed its analogues owing to its more ordered and long-range molecular packing and better hostdopant miscibility. P2G showed lower electrical conductivity values, with good host-dopant miscibility, but no long-range molecular packing. Finally, N-type doped P3G films showed the lowest conductivity of the three polymers due to its amorphous nature and poor host-dopant miscibility. This study showcases the impact of the backbone orientation on the efficiency of molecular doping and in the miscibility of molecular dopants where low polarity and miscibility of P3G overcame the presence of branched TEG side chains, limiting molecular doping with N-DMBI. Finally, the thermoelectric properties of P1G showed a maximum power factor at $5 \mathrm{~mol} \%$ of $\mathrm{N}$-DMBI, with a particularly low in-plane thermal conductivity. We believe this low value is enabled by the disordered nature of the branched TEG side chains, which opens a new path to discovering new conjugated polymers with ultra-low thermal conductivity values. 


\section{Conflicts of interest}

The authors declare no conflicts of interest.

\section{Acknowledgements}

This publication is based upon work supported by the King Abdullah University of Science and Technology (KAUST) Office of Sponsored Research (OSR) under Award No. OSR-CRG20183737 and British Council Newton Fund Institutional Links (ref: 337067). B.C.S. acknowledges the UK Research and Innovation for Future Leaders Fellowship no. MR/S031952/1 and EPSRC grant (EP/P007767/1). L. G. and X. G. thanks U.S. Department Energy provides partial support for the scattering experiments in this work under award No. DE-SC0022050. The work by J. L. and L. J. A. K. was supported by a grant from STW/NWO (VIDI 13476).

\section{References}

1 F. Zhang, Y. Zang, D. Huang, C. A. Di and D. Zhu, Nat. Commun., 2015, 6, 8356.

2 R. Kroon, D. A. Mengistie, D. Kiefer, J. Hynynen, J. D. Ryan, L. Yu and C. Muller, Chem. Soc. Rev., 2016, 45, 6147-6164.

3 O. Bubnova and X. Crispin, Energy Environ. Sci., 2012, 5, 9345-9362.

4 I. E. Jacobs and A. J. Moule, Adv. Mater., 2017, 29, 1703063.

5 Y. Xu, H. Sun, A. Liu, H. H. Zhu, W. Li, Y. F. Lin and Y. Y. Noh, Adv. Mater., 2018, 30, e1801830.

6 B. Russ, A. Glaudell, J. J. Urban, M. L. Chabinyc and R. A. Segalman, Nat. Rev. Mater., 2016, 1, 16050.

7 L. M. Cowen, J. Atoyo, M. J. Carnie, D. Baran and B. C. Schroeder, ECS J. Solid State Sci. Technol., 2017, 6, N3080-N3088.

8 W. Zhao, J. Ding, Y. Zou, C. A. Di and D. Zhu, Chem. Soc. Rev., 2020, 49, 7210-7228.

9 X. Peng, L. Hu, F. Qin, Y. Zhou and P. K. Chu, Adv. Mater. Interfaces, 2018, 5, 1701404.

10 A. F. Paterson, A. Savva, S. Wustoni, L. Tsetseris, B. D. Paulsen, H. Faber, A. H. Emwas, X. Chen, G. Nikiforidis, T. C. Hidalgo, M. Moser, I. P. Maria, J. Rivnay, I. McCulloch, T. D. Anthopoulos and S. Inal, Nat. Commun., 2020, 11, 3004.

11 M. Kang, S.-M. Ko, J. Kim, S. Z. Hassan, D.-W. Jee and D. S. Chung, Mater. Horiz., 2020, 7, 3034-3042.

12 O. Bubnova, Z. U. Khan, A. Malti, S. Braun, M. Fahlman, M. Berggren and X. Crispin, Nat. Mater., 2011, 10, 429-433.

13 V. Vijayakumar, Y. Zhong, V. Untilova, M. Bahri, L. Herrmann, L. Biniek, N. Leclerc and M. Brinkmann, Adv. Energy Mater., 2019, 9, 1900266.

14 C. Y. Yang, Y. F. Ding, D. Huang, J. Wang, Z. F. Yao, C. X. Huang, Y. Lu, H. I. Un, F. D. Zhuang, J. H. Dou, C. A. Di, D. Zhu, J. Y. Wang, T. Lei and J. Pei, Nat. Commun., 2020, 11, 3292.

15 M. Xiong, X. Yan, J. T. Li, S. Zhang, Z. Cao, N. Prine, Y. Lu, J. Y. Wang, X. Gu and T. Lei, Angew. Chem., Int. Ed., 2021, 60, 8189-8197.
16 J. Liu, B. van der Zee, R. Alessandri, S. Sami, J. Dong, M. I. Nugraha, A. J. Barker, S. Rousseva, L. Qiu, X. Qiu, N. Klasen, R. C. Chiechi, D. Baran, M. Caironi, T. D. Anthopoulos, G. Portale, R. W. A. Havenith, S. J. Marrink, J. C. Hummelen and L. J. A. Koster, Nat. Commun., 2020, 11, 5694.

17 M. Goel, C. D. Heinrich, G. Krauss and M. Thelakkat, Macromol. Rapid Commun., 2019, 40, e1800915.

18 F. Zhang and C.-a. Di, Chem. Mater., 2020, 32, 2688-2702. 19 H. Jia and T. Lei, J. Mater. Chem. C, 2019, 7, 12809-12821. 20 J. Liu, L. Qiu, R. Alessandri, X. Qiu, G. Portale, J. Dong, W. Talsma, G. Ye, A. A. Sengrian, P. C. T. Souza, M. A. Loi, R. C. Chiechi, S. J. Marrink, J. C. Hummelen and L. J. A. Koster, Adv. Mater., 2018, 30, 1704630.

21 D. Kiefer, A. Giovannitti, H. Sun, T. Biskup, A. Hofmann, M. Koopmans, C. Cendra, S. Weber, L. J. Anton Koster, E. Olsson, J. Rivnay, S. Fabiano, I. McCulloch and C. Muller, ACS Energy Lett., 2018, 3, 278-285.

22 J. Liu, G. Ye, H. G. O. Potgieser, M. Koopmans, S. Sami, M. I. Nugraha, D. R. Villalva, H. Sun, J. Dong, X. Yang, X. Qiu, C. Yao, G. Portale, S. Fabiano, T. D. Anthopoulos, D. Baran, R. W. A. Havenith, R. C. Chiechi and L. J. A. Koster, Adv. Mater., 2021, 33, e2006694.

23 J. Liu, L. Qiu, G. Portale, S. Torabi, M. C. A. Stuart, X. Qiu, M. Koopmans, R. C. Chiechi, J. C. Hummelen and L. J. Anton Koster, Nano Energy, 2018, 52, 183-191.

24 S. Wang, H. Sun, T. Erdmann, G. Wang, D. Fazzi, U. Lappan, Y. Puttisong, Z. Chen, M. Berggren, X. Crispin, A. Kiriy, B. Voit, T. J. Marks, S. Fabiano and A. Facchetti, Adv. Mater., 2018, 30, e1801898.

25 J. Liu, G. Ye, B. V. Zee, J. Dong, X. Qiu, Y. Liu, G. Portale, R. C. Chiechi and L. J. A. Koster, Adv. Mater., 2018, 30, e1804290.

26 K. Shi, F. Zhang, C. A. Di, T. W. Yan, Y. Zou, X. Zhou, D. Zhu, J. Y. Wang and J. Pei, J. Am. Chem. Soc., 2015, 137, 6979-6982.

27 C. Y. Yang, W. L. Jin, J. Wang, Y. F. Ding, S. Nong, K. Shi, Y. Lu, Y. Z. Dai, F. D. Zhuang, T. Lei, C. A. Di, D. Zhu, J. Y. Wang and J. Pei, Adv. Mater., 2018, 30, e1802850.

28 J. Liu, L. Qiu, G. Portale, M. Koopmans, G. Ten Brink, J. C. Hummelen and L. J. A. Koster, Adv. Mater., 2017, 29, 1701641.

29 A. Sharma, S. Singh, X. Song, D. Rosas Villalva, J. Troughton, D. Corzo, L. Toppare, G. Gunbas, B. C. Schroeder and D. Baran, Chem. Mater., 2021, 33, 8602-8611.

30 B. D. Naab, X. Gu, T. Kurosawa, J. W. F. To, A. Salleo and Z. Bao, Adv. Electron Mater., 2016, 2, 1600004.

31 T. Lei, J. H. Dou, X. Y. Cao, J. Y. Wang and J. Pei, J. Am. Chem. Soc., 2013, 135, 12168-12171.

32 H. Huang, L. Yang, A. Facchetti and T. J. Marks, Chem. Rev., 2017, 117, 10291-10318.

33 Y. Wang, T. Hasegawa, H. Matsumoto and T. Michinobu, J. Am. Chem. Soc., 2019, 141, 3566-3575.

34 T. Erdmann, S. Fabiano, B. Milian-Medina, D. Hanifi, Z. Chen, M. Berggren, J. Gierschner, A. Salleo, A. Kiriy, B. Voit and A. Facchetti, Adv. Mater., 2016, 28, 9169-9174. 
35 Y. Kim, H. Hwang, N. K. Kim, K. Hwang, J. J. Park, G. I. Shin and D. Y. Kim, Adv. Mater., 2018, 30, e1706557.

36 M. Moser, A. Savva, K. Thorley, B. D. Paulsen, T. C. Hidalgo, D. Ohayon, H. Chen, A. Giovannitti, A. Marks, N. Gasparini, A. Wadsworth, J. Rivnay, S. Inal and I. McCulloch, Angew. Chem., Int. Ed., 2021, 60, 7777-7785.

37 T. Lei, X. Xia, J. Y. Wang, C. J. Liu and J. Pei, J. Am. Chem. Soc., 2014, 136, 2135-2141.

38 L. Gao, Z. G. Zhang, L. Xue, J. Min, J. Zhang, Z. Wei and Y. Li, Adv. Mater., 2016, 28, 1884-1890.

39 M. Koopmans, M. A. T. Leiviska, J. Liu, J. Dong, L. Qiu, J. C. Hummelen, G. Portale, M. C. Heiber and L. J. A. Koster, ACS Appl. Mater. Interfaces, 2020, 12, 56222-56230.
40 L. Qiu, J. Liu, R. Alessandri, X. Qiu, M. Koopmans, R. W. A. Havenith, S. J. Marrink, R. C. Chiechi, L. J. Anton Koster and J. C. Hummelen, J. Mater. Chem. A, 2017, 5, 21234-21241.

41 J. Sworakowski, Synth. Met., 2018, 235, 125-130.

42 Z. Liang, H. H. Choi, X. Luo, T. Liu, A. Abtahi, U. S. Ramasamy, J. A. Hitron, K. N. Baustert, J. L. Hempel, A. M. Boehm, A. Ansary, D. R. Strachan, J. Mei, C. Risko, V. Podzorov and K. R. Graham, Nat. Mater., 2021, 20, 518-524.

43 S. Kommandur and S. Yee, Rev. Sci. Instrum., 2018, 89, 114905. 44 D. Scheunemann and M. Kemerink, Phys. Rev. B, 2020, 101, 075206. 